\title{
Characterization of Plum Procyanidins by Thiolytic Depolymerization
}

\author{
Cláudia Nunes, ${ }^{\dagger}$ Sylvain Guyot, ${ }^{\ddagger}$ Nathalie Marnet, ${ }^{\ddagger}$ António S. Barros, ${ }^{\dagger}$ \\ Jorge A. Saraiva, ${ }^{\dagger}$ Catherine M. G. C. Renard, ${ }^{\S}$ AND \\ MANuEl A. COIMBRA*, ${ }^{*}$ \\ Departamento de Química, Universidade de Aveiro, 3810-193 Aveiro, Portugal, INRA, UR117, \\ Recherches Cidricoles et Biotransformation des Fruits et Légumes, F-35653 Le Rheu, France, and \\ UMR 408 SQPOV, INRA, Domaine Saint Paul, 84914 Avignon Cedex 9, France
}

\begin{abstract}
The phenolic compounds of 'Green Gage' (GG) plums (Prunus domestica L.), "Rainha Cláudia Verde”, from a 'protected designation of origin' (PDO), in Portugal, were quantified in both flesh and skin tissues of plums collected in two different orchards (GG-V and GG-C). Analyzes of phenolic compounds were also performed on another GG European plum obtained in France (GG-F) and two other French plums, 'Mirabelle' (M) and 'Golden Japan' (GJ). Thiolysis was used for the first time in the analysis of plum phenolic compounds. This methodology showed that the flesh and skin contain a large proportion of flavan-3-ols, which account, respectively, for 92 and $85 \%$ in GJ, 61 and $44 \%$ in GG-V, 62 and $48 \%$ in GG-C, 54 and $27 \%$ in M, and 45 and $37 \%$ in GG-F. Terminal units of procyanidins observed in plums are mainly $(+)$-catechin $(54-77 \%$ of all terminal units in flesh and $57-81 \%$ in skin). The GJ plums showed a phenolic composition different from all of the others, with a lower content of chlorogenic acid isomers and the presence of A-type procyanidins as dimers and terminal residues of polymerized forms. The average degree of polymerization (DPn) of plum procyanidins was higher in the flesh (5-9 units) than in the skin (4-6 units). Procyanidin B7 was observed in the flesh of all GG plums and in the skin of the Portuguese ones. Principal component analysis of the phenolic composition of the flesh and skin of these plums obtained after thiolysis allowed their distinction according to the variety and origin, opening the possibility of the use of phenolic composition for variety/origin identification.
\end{abstract}

KEYWORDS: 'Green Gage'; 'Mirabelle’; ‘Golden Japan'; reversed-phase HPLC; phenolic compounds; hydroxycinnamic acid; flavan-3-ols; flavonols

\section{INTRODUCTION}

Phenolic compounds are relevant for the establishment of fruit quality, because they play a role in the visual appearance (pigmentation and browning), taste (astringency), and healthpromoting properties (free-radical scavengers) of fresh fruits and their derived products $(1-3)$. Some of the health benefits related to fruit consumption seem to be due to the content of phenolic compounds with antioxidant, anticarcinogenic, antimicrobial, antiallergic, antimutagenic, and anti-inflammatory activities $(1,2,4)$. As a general rule, accumulation of soluble phenolic compounds in fruits is greater in the outer tissues (skin) than in the inner tissues (flesh) $(1,5)$. The level and composition of phenolic compounds in plums are of great importance for some

* To whom correspondence should be addressed. Telephone: +351 234-370706. Fax: +351-234-370084. E-mail: mac@ua.pt.

$\dagger$ Universidade de Aveiro.

*INRA, UR117, Recherches Cidricoles et Biotransformation des Fruits et Légumes.

${ }^{\S}$ UMR 408 SQPOV, INRA. of the health-promoting benefits attributed to the consumption of these fruits. For instance, studies measuring the total antioxidant capacity of a number of fruits revealed that plums showed one of the highest antioxidant activities within Mediterranean fruits (2, 6-9). Among the molecules exerting antioxidant activity in plums, the contribution of phenolic compounds was found to be much greater than vitamin $\mathrm{C}$ and carotenoids $(2,7,10-12)$.

The predominant class of phenolic compounds identified in plums was the hydroxycinnamic acid derivatives, such as 3-caffeoylquinic acid (neochlorogenic acid) and 5-caffeoylquinic acid (chlorogenic acid), with the former being present in higher amounts (13-15). Flavan-3-ols were also identified in plums, predominantly the procyanidin dimers of the B and A types, as well as procyanidin trimers and monomers (13). Rutin (quercetin 3 -rutinoside) was the principal polyphenol among the flavonol glycosides found in plums (13). However, no information is yet available about the total concentration and degree of polymerization of plum procyanidins. 
The presence of flavan-3-ol monomers and procyanidins in the phenolic composition of the fruits is of great importance for their nutritional and functional properties. The monomers, which are good substrates for polyphenol oxidase, are directly involved in enzymatic oxidation. Conversely, procyanidins could be involved in oxidation reactions (16). Flavan-3-ols could have several physiological roles, as protective against fungi attacks and biological properties, with these properties being directly correlated with the molecular weight of procyanidins (17-19). A-type dimers have been reported as having antibacterial activity (14).

Protected designation of origin (PDO) is an official label used in the European Union for food products that are produced, processed, and prepared in a given geographical area, because distinctive quality and organoleptic characteristics. Despite this, scientific information available to support the unique quality of many PDO products, although essential, is still very scarce, as is the case of the 'Ameixa d'Elvas' plum.

'Ameixa d'Elvas' is a PDO for a plum (Prunus domestica L.) of a specific type of 'Green Gage' variety, "Rainha Cláudia Verde", produced in a defined region in Alto Alentejo (southeast of Portugal). The aim of this work was to characterize these plums by their phenolic composition, for possible further use of the required information to unambiguously identify these plums. For this objective, the phenolic composition of PDO 'Green Gage' plums from Portugal (collected in two different orchards from the same limited geographic area within the PDO) was studied together with the plums of the same variety but from a different geographic origin (France) and also with plums of other variety and specie ('Mirabelle' and 'Golden Japan'), whose phenolic composition has not yet been established. Reversed-phase high-performance liquid chromatography (RPHPLC) analyses were performed on crude methanolic extracts from both the skin and flesh. Additionally, RP-HPLC were also carried out after thiolysis on freeze-dried materials of skin and flesh to obtain more information on the total procyanidin content with global characterization of their average degree of polymerization and their constitutive flavanol units.

\section{MATERIALS AND METHODS}

Solvents and Reagents. Methanol, formic acid, and acetonitrile of chromatographic-grade quality and glacial acetic acid were purchased from Biosolve Ltd. (Netherlands). Folin-Ciocalteu reagent was purchased from Merck (Germany). Deionized water was obtained with a Milli-Q water system (Millipore Corporation, Bedford, MA).

Phenolic Standards. (+)-Catechin, (-)-epicatechin, 5-caffeoylquinic acid, and rutin were provided by Sigma-Aldrich, Inc. (Bellefonte, PA). Hyperoside (quercetin 3-galactoside), isoquercitrin (quercetin 3-glucoside), quercitrin (quercetin 3-rhamnoside), myricetin, and isorhamnetin rutinoside were provided by Extrasynthese S.A. (France). (-)Epicatechin benzylthioether was obtained as described by Guyot et al. (20). Procyanidin A2 was kindly provided by E. Meudec (UMR-SPO, INRA, Montpellier, France). Procyanidins B1, B2, and B7 were available at INRA, UR117 (Le Rheu, France) as standards isolated from apple for B2 or by hemisynthesis for B1 and B7, according to a method adapted from previously published results of Hemingway and McGraw (21).

Samples. Plums (Prunus domestica L.) of 'Green Gage' variety were harvested at a mature stage in 2006 in two orchards from Alto Alentejo, Portugal ('Cano', GG-C; 'Vila Viçosa', GG-V). The analyzes were also performed on three varieties of French plums, 'Green Gage' (GG-F) (Prunus domestica L.), 'Mirabelle' (M) (Prunus domestica L.), and 'Golden Japan' (GJ) (Prunus salicina Lindl.) obtained in a local market in Rennes (France). All plums were brought to the laboratory and immediately prepared for analysis.

Plums (1 kg) were peeled, and the flesh and skin were separately immersed in a $\mathrm{NaF}$ solution (to inhibit oxidation) at $1 \mathrm{~g} / \mathrm{L}$ in water, with a proportion of $2 \mathrm{~g}$ of fresh material for $3 \mathrm{~mL}$ of aqueous solution, and homogenized in a Waring blender for $1 \mathrm{~min}$. The homogenized samples were immediately frozen and freeze-dried.

Extraction of Phenolic Compounds. Phenolic compounds were extracted from freeze-dried plum samples $(0.1 \mathrm{~g})$ with $1 \mathrm{~mL}$ of methanol (20) containing $1 \%$ acetic acid (to avoid oxidation) using an ultrasoundassisted method (22) for $15 \mathrm{~min}$, at room temperature. This crude methanol extract was filtered through a $0.45 \mu \mathrm{m}$ filter (HV, Millipore Corporation, Bedford, MA) and immediately analyzed. Three extractions were carried out for each sample.

Quantification of Total Phenolic Compounds. Total phenolic compounds were quantified by the colorimetric method of Folin-Ciocalteu (23), adapted from Guyot et al. (20). The crude methanol extract was properly diluted with acetic acid $[2 \%(\mathrm{v} / \mathrm{v})$ in water], and $0.5 \mathrm{~mL}$ of extract was added to $250 \mu \mathrm{L}$ of Folin-Ciocalteu reagent. This procedure, when applied to plums, according to Chun and Kim (24), does not require correction for vitamin $\mathrm{C}$ or reducing sugars content.

5-Caffeoylquinic acid and (-)-epicatechin were both used as standards. The calibration curves were performed with seven different concentrations, and each one was measured in triplicate. Quantifications of phenolic compounds were obtained by reporting the absorbances in both calibration curves of 5 -caffeoylquinic acid $(0.02-0.12 \mathrm{mg} / \mathrm{mL})$ and (-)-epicatechin $(0.02-0.17 \mathrm{mg} / \mathrm{mL})$. Phenolic compounds in each extract were quantified in triplicate.

Thiolysis of the Samples. The thiolysis of plum flesh and skin was performed on $50 \mathrm{mg}$ of homogeneous freeze-dried powder with 800 $\mu \mathrm{L}$ of benzylthiolether [5\% (v/v) in dry methanol] and $400 \mu \mathrm{L}$ of 0.4 $\mathrm{M} \mathrm{HCl}$ in dry methanol (25), and the reaction was carried out at $40^{\circ} \mathrm{C}$ during $30 \mathrm{~min}$. The samples were filtered through a $0.45 \mu \mathrm{m}$ Teflon membrane (Millipore, Bedford, MA) and then analyzed. Thiolysis reactions were performed in triplicate for each sample.

RP-HPLC. The crude methanol extracts and the thiolysis reaction mixtures were analyzed by RP-HPLC. The HPLC apparatus was a Waters (Milford, MA) system, equipped with an autosampler 717, with a cooling module set at $4{ }^{\circ} \mathrm{C}$, a $600 \mathrm{E}$ multisolvent pump delivery system, and a 996 photodiode array detector. The data acquisition and remote control of the HPLC system was performed by the Millennium 32 version 3.20 software. The column was a Fusion-RP, polarembedded C18 (Synergi, Phenomenex, Inc., Torrance, CA), and the eluents used were $1 \%$ formic acid in water (solvent $\mathrm{A}$ ) and acetonitrile (solvent B), degassed continuously with helium. The flow rate was 1 $\mathrm{mL} / \mathrm{min}$, and the injection volume was $5 \mu \mathrm{L}$. The solvent gradient started with $95 \%$ A; 0-34 min, $60 \%$ A linear; $34-37$ min, $10 \%$ A linear; 37-42 min, 10\% A isocratic; 42-47 min, 95\% A linear; and returned to initial conditions. The column oven was set at $35^{\circ} \mathrm{C}$. The eluate was continuously monitored between 190 and $600 \mathrm{~nm}$. Phenolic compounds in the crude extracts and thiolysed samples were quantified in triplicate.

RP-HPLC Characterization and Quantification of Phenolic Compounds. Phenolic compounds were identified on chromatograms by comparison of their retention times with available standards and UV-vis spectra: caffeic acid, (+)-catechin, 5-caffeoylquinic acid, $p$-coumaric acid, $p$-coumaroylquinic acid, (-)-epicatechin, (-)-epicatechin benzylthioether, ferulic acid, hyperoside (quercetin 3-galactoside), isoquercitrin (quercetin 3-glucoside), isorhamnetin rutinoside, kaempferol glucoside, kaempferol rutinoside, myricetin, phloretin, procyanidins A, B1, B2, B5, and B7, quercetin, quercitrin (quercetin 3-rhamnoside), and rutin. Because of the unavailability of authentic commercial 3-caffeoylquinic acid standard, its identification was accomplished by comparison of a previously reported HPLC separation pattern and its $\mathrm{UV}-\mathrm{vis}$ spectrum with that of 5-caffeoylquinic acid $(13,14,26)$. Although other compounds could not be completely identified, they were characterized according to their class on the basis of their UV-vis spectra (27): flavan-3-ols (monomers and procyanidins) by the presence of a single symmetric band with a maximum absorbance at $278 \mathrm{~nm}$, hydroxycinnamic acid derivatives that presented a maximum absorbance between 300 and $330 \mathrm{~nm}$, and flavonols that showed an absorption band with a maximum above $340 \mathrm{~nm}$.

Quantification of the identified compounds was performed using external calibration curves obtained for $(+)$-catechin $(0.01-0.14 \mathrm{mg} /$ $\mathrm{mL})$, (-)-epicatechin $(0.02-0.10 \mathrm{mg} / \mathrm{mL}), 5$-caffeoylquinic acid 
Table 1. Average Fresh Weight of Intact Plums and Relative Proportion of the Flesh, Skin, and Stone ${ }^{a}$

\begin{tabular}{|c|c|c|c|c|c|c|}
\hline plum varieties & weight $(\mathrm{g})$ & flesh (\%) & flesh moisture (\%) & skin (\%) & skin moisture (\%) & stone $(\%)$ \\
\hline GG-V & $38 \pm 3$ & 74 & $83 \pm 1$ & 16 & $76 \pm 1$ & 10 \\
\hline GG-C & $31 \pm 1$ & 72 & $82 \pm 0$ & 18 & $76 \pm 1$ & 10 \\
\hline GG-F & $26 \pm 3$ & 72 & $89 \pm 1$ & 16 & $89 \pm 1$ & 12 \\
\hline $\mathrm{M}$ & $10 \pm 2$ & 80 & $89 \pm 1$ & 11 & $91 \pm 0$ & 9 \\
\hline GJ & $55 \pm 7$ & 66 & $94 \pm 0$ & 25 & $92 \pm 0$ & 9 \\
\hline
\end{tabular}

a GG-V, ‘Green Gage'-Portugal from “Vila Viçosa” orchard; GG-C, 'Green Gage'-Portugal from “Cano” orchard; GG-F, ‘Green Gage'-France; GJ, 'Golden Japan'; M, 'Mirabelle'. Mean \pm standard deviation $(n=10)$.

$(0.02-0.9 \mathrm{mg} / \mathrm{mL})$, rutin $(0.02-0.2 \mathrm{mg} / \mathrm{mL})$, and (-)-epicatechin benzylthioether $(0.02-0.5 \mathrm{mg} / \mathrm{mL})$. The quantification of hydroxycinnamic acid derivatives was evaluated at $320 \mathrm{~nm}$ applying the 5-caffeoylquinic acid calibration curve. The flavonols were quantified at 350 $\mathrm{nm}$ using rutin as an external standard.

The average degree of polymerization (DPn) of flavanols (including monomeric catechins plus procyanidins) was calculated as previously published for apple samples (20). For 'Golden Japan' variety that contained A-type moieties in the procyanidin structure, the DPn was calculated by taking into account A-type dimers formed in thiolysis media according to the formula given by Le Roux et al. (28).

HPLC-Electrospray Ionization-Mass Spectrometry (ESI-MS). HPLC-ESI-MS was used for unambiguous detection of phenolic compounds in the samples, to eliminate misidentification of coeluting compounds with similar UV spectra. The MS system was a LCQ Deca ion trap (Thermofinnigan, San Jose, CA) equipped with an ESI source. ESI-MS was coupled to a HPLC system consisting of a degasser spectra system SMC 1000 (Thermofinnigan, San Jose, CA), a HP quaternary gradient Pump 1100 series (Agilent Technologies), an autosampler Surveyor (Thermofinnigan, San Jose, CA), a Zorbax Eclipse XDBC18 column $(2.1 \mathrm{~cm} \times 150 \mathrm{~mm})$, a diode array detector (DAD) spectra system UV 6000 LP (Thermofinnigan, San Jose, CA), and Xcalibur version 1.2 software to monitor the system and acquire and process the data. The crude extracts and thiolysed samples were injected onto the HPLC-ESI-MS system. The eluting conditions were as follows: solvent $\mathrm{A}$ ( $0.1 \%$ formic acid in purified water), solvent $\mathrm{B}$ (acetonitrile with $0.1 \%$ formic acid), the flow was set at $0.2 \mathrm{~mL} / \mathrm{min}$, and the applied gradient was 3\% B; 0-5 min, 9\% B linear; 5-15 min,
$16 \%$ B linear; $15-45 \mathrm{~min}, 50 \%$ B; then 5 min of washing with $90 \%$ of $\mathrm{B}$; and a return to initial conditions for equilibration. The mass spectrometer was used in the negative mode, and the source parameters were spray voltage of $4500 \mathrm{kV}$, orifice voltage of $-60 \mathrm{~V}$, capillary temperature of $225^{\circ} \mathrm{C}$, a 50 arbitrary units sheath nitrogen gas flow rate. Ions were detected in the $\mathrm{m} / \mathrm{z}, 50-2000$ range.

Principal Component Analysis (PCA). PCA (29) was applied to the compounds identified before thiolysis for hydroxycinnamic acids and flavonols and after thiolysis for flavan-3-ols using the values on a $\mathrm{mg} / \mathrm{kg}$ fresh weight basis given in Tables $\mathbf{2}$ and $\mathbf{3}$. The flesh data matrix comprised 5 samples of the plums flesh ( 3 repetitions) and 12 phenolic parameters, giving a matrix with 15 rows and 12 columns. For skin data, the same plum samples were used, but this time, 18 phenolic parameters were measured, thus giving a matrix with 15 rows and 18 columns. The only preprocessing method used was centering the matrices by column before PCA.

Statistical Analysis. Results are presented as mean values, and the reproducibility of the results was expressed as standard deviation in the tables and error bars in the figures. Statistical analysis between experimental results was based on Student's $t$ test. Significant difference was statistically considered at the level of $p<0.05$.

\section{RESULTS AND DISCUSSION}

Two different tissue zones of the plums were considered for the analysis of their polyphenolic content, the epidermis (skin) and the parenchyma (flesh). The fruits analyzed had different average weight, and the relative amount of skin and flesh was
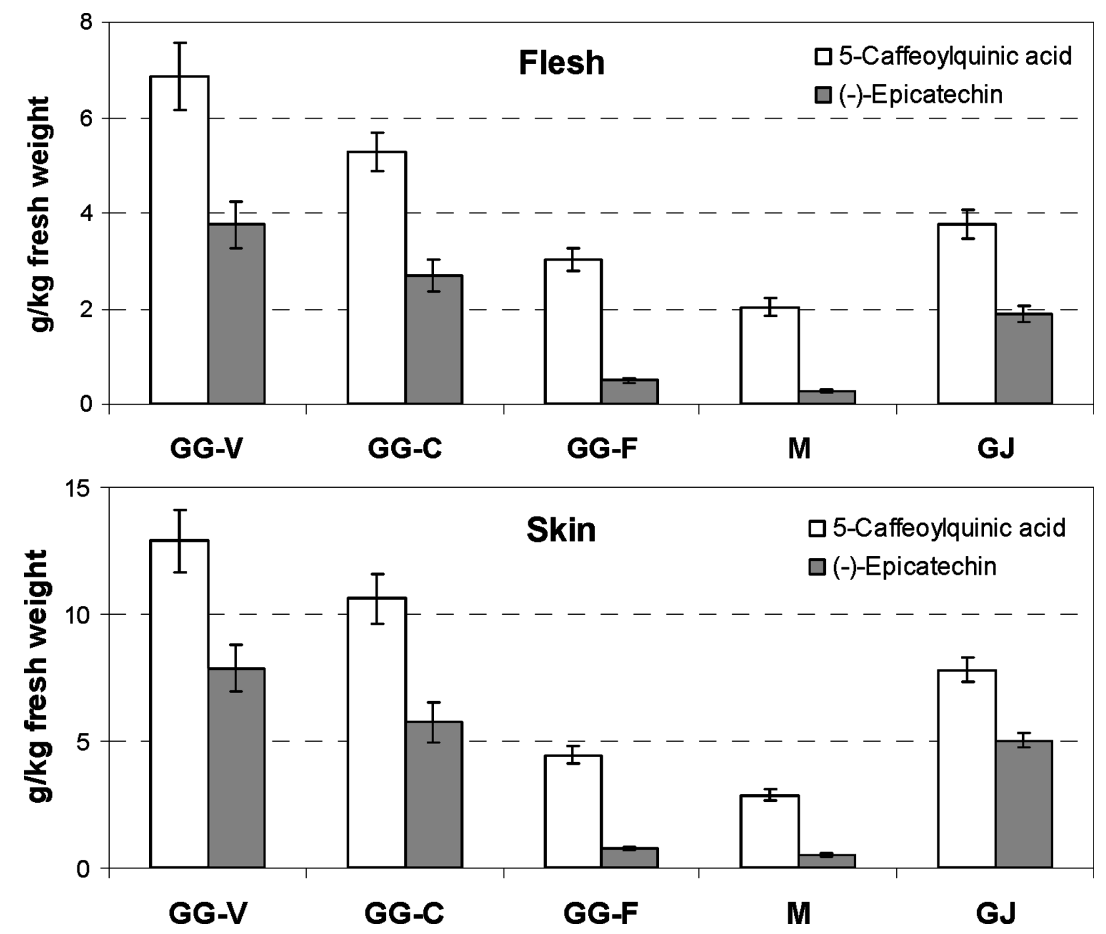

Figure 1. Total phenolic compounds of plum methanolic extracts by the Folin-Ciocalteu method of the flesh and skin (mg/fruit), expressed as 5-caffeoylquinic acid and (-)-epicatechin (GG-C, 'Green Gage'-Portugal "Cano”; GG-F, 'Green Gage'-France; GG-V, 'Green Gage'-Portugal "Vila Viçosa”; GJ, 'Golden Japan'; M, 'Mirabelle'). 
Table 2. Quantification of Phenolic Compounds in Plum Flesh (mg/kg of Fresh Weight and mg/Fruit) by RP-HPLC, for Crude Extract and after Thiolysis ${ }^{a}$

\begin{tabular}{|c|c|c|c|c|c|c|c|c|c|c|}
\hline & \multicolumn{2}{|c|}{ GG-V } & \multicolumn{2}{|c|}{ GG-C } & \multicolumn{2}{|c|}{ GG-F } & \multicolumn{2}{|c|}{$M$} & \multicolumn{2}{|c|}{ GJ } \\
\hline \multicolumn{11}{|c|}{ Hydroxycinnamic Acids } \\
\hline 5CQA (Rt $15.5 \mathrm{~min})$ & $162 \pm 7$ & 5 & $163 \pm 7$ & 4 & $165 \pm 7$ & 3 & $134 \pm 5$ & 1 & $27 \pm 1$ & 1 \\
\hline $\mathrm{FQA}^{b}$ (Rt $\left.15.0 \mathrm{~min}\right)$ & & & & & & & $35 \pm 3$ & 1 & & \\
\hline $\mathrm{CSA}^{b}$ (Rt $\left.13.9 \mathrm{~min}\right)$ & $65 \pm 4$ & 2 & $68 \pm 3$ & 2 & $97 \pm 5$ & 2 & $42 \pm 2$ & 1 & $20 \pm 1$ & 1 \\
\hline unknown (Rt $20.5 \mathrm{~min}$ ) & $28 \pm 3$ & 1 & $30 \pm 2$ & 1 & $34 \pm 1$ & 1 & & & & \\
\hline unknown (Rt $22.3 \mathrm{~min}$ ) & $30 \pm 3$ & 1 & $30 \pm 2$ & 1 & $43 \pm 2$ & 1 & & & & \\
\hline subtotal & 2005 & 57 & 2128 & 49 & 2570 & 49 & 1188 & 12 & 200 & 8 \\
\hline \multicolumn{11}{|c|}{ Flavan-3-ols } \\
\hline B1 (Rt 13.4 min) & $183 \pm 14$ & 5 & $140 \pm 5$ & 3 & $58 \pm 3$ & 1 & $54 \pm 5$ & 1 & $276 \pm 10$ & 10 \\
\hline CAT (Rt $14.8 \mathrm{~min}$ ) & $121 \pm 3$ & 3 & $119 \pm 5$ & 3 & $68 \pm 3$ & 1 & $15 \pm 3$ & $\mathrm{tr}$ & $220 \pm 10$ & 8 \\
\hline unknown (Rt $14.3 \mathrm{~min}$ ) & $39 \pm 4$ & 1 & $34 \pm 1$ & 1 & & & & & $86 \pm 3$ & 3 \\
\hline unknown (Rt $15.8 \mathrm{~min}$ ) & $55 \pm 3$ & 2 & $29 \pm 1$ & 1 & & & & & $64 \pm 2$ & 2 \\
\hline unknown (Rt $16.8 \mathrm{~min}$ ) & $39 \pm 2$ & 1 & $40 \pm 2$ & 1 & & & & & $28 \pm 1$ & 1 \\
\hline unknown (Rt $18.9 \mathrm{~min}$ ) & $42 \pm 6$ & 1 & $33 \pm 1$ & 1 & & & & & $33 \pm 1$ & 1 \\
\hline unknown (Rt $20.2 \mathrm{~min}$ ) & $42 \pm 6$ & 1 & $36 \pm 2$ & 1 & & & & & $62 \pm 6$ & 2 \\
\hline unknown (Rt $21.1 \mathrm{~min}$ ) & $50 \pm 7$ & 1 & $55 \pm 3$ & 1 & $54 \pm 4$ & 1 & $33 \pm 3$ & $\operatorname{tr}$ & $34 \pm 7$ & 1 \\
\hline unknown (Rt $23.3 \mathrm{~min}$ ) & & & & & & & $38 \pm 2$ & $\operatorname{tr}$ & $65 \pm 12$ & 2 \\
\hline subtotal & 781 & 21 & 655 & 16 & 242 & 5 & 169 & 1 & 1133 & 40 \\
\hline total crude extract & 2786 & 78 & 2783 & 65 & 2812 & 54 & 1357 & 13 & 1333 & 48 \\
\hline \multicolumn{11}{|c|}{ Procyanidins } \\
\hline CAT & $395 \pm 10$ & 11 & $497 \pm 17$ & 11 & $314 \pm 14$ & 6 & $194 \pm 6$ & 2 & $601 \pm 28$ & 22 \\
\hline $\begin{array}{l}\mathrm{EC} \\
\mathrm{A}\end{array}$ & $160 \pm 4$ & 4 & $198 \pm 9$ & 4 & $137 \pm 8$ & 3 & $123 \pm 1$ & 1 & $\begin{array}{c}50 \pm 2 \\
242 \pm 13\end{array}$ & $\begin{array}{l}2 \\
9\end{array}$ \\
\hline
\end{tabular}

${ }^{a}$ Flavan-3-ols are expressed as catechin equivalents, and hydroxycinnamic acids are expressed as 5-caffeoylquinic acid equivalents. A, A-type procyanidin, B1, procyanidin B1; B2, procyanidin B2; B7, procyanidin B7; CAT, (+)-catechin; 3CQA, 3-caffeoylquinic acid; 5CQA, 5-caffeoylquinic acid; CSA, caffeoylshikimic acid; DPn, average degree of polymerization; EC, (-)-epicatechin; EC-Ext, (-)-epicatechin benzylthioether; FQA, feruloylquinic acid; GG-C, 'Green Gage'-Portugal "Cano"; GG-F, 'Green Gage'France; GG-V, 'Green Gage'-Portugal "Vila Viçosa”; GJ, 'Golden Japan'; M, 'Mirabelle'; Rt, retention time, tr, trace amounts, $<0.5 \mathrm{mg} /$ fruit. Mean \pm standard deviation ( $n$ $=3) .{ }^{b}$ Identification only by ESI-MS, no standard available.

also different, as showed in Table $\mathbf{1}$. The GJ plums were bigger $(55 \mathrm{~g})$ and had the highest amount of skin (25\%) when compared to the other plums, while the $\mathrm{M}$ variety had the smallest fruits $(10 \mathrm{~g})$ and the lowest amount of skin $(11 \%)$. The weight of GG varied from $26 \mathrm{~g}$ (GG-F) to $38 \mathrm{~g}(\mathrm{GG}-\mathrm{V})$, and the skin amount ranged from $16-18 \%$.

Quantification of Total Phenolic Compounds by the Folin-Ciocalteu Method. The results obtained for the total phenolic compounds recovered from the flesh and skin of all plum varieties are presented in Figure 1. Two standard curves, 5-caffeoylquinic acid (5CQA) and (-)-epicatechin (EC), were performed in this study because the quantification of total phenolics depends upon the standard used.

The values obtained using the two standards show a higher estimation when 5CQA was used as compared to EC, for both flesh and skin. This is in agreement with the fact that 5CQA shows the lowest response factor, using the Folin-Ciocalteau reagent, compared to other phenolic standards $(23,24)$. In the present study, the ratio between the values of total phenolics calculated using EC and 5CQA as standards was not the same for all samples. Plums of $\mathrm{M}$ and GG-F varieties showed a higher difference $(83-86 \%)$ than the other plums $(36-50 \%)$.

The skin showed a significantly higher amount of phenolic compounds (1.5-2-fold) compared to the flesh tissue for all samples analyzed (Figure 1), which is in accordance with studies for other plum varieties $(13,30)$. The different varieties showed a wide variation on total phenolics content, for both flesh and skin. Total phenolic content ranged from 2.0 to $6.8 \mathrm{~g}$ of $5 \mathrm{CQA} / \mathrm{kg}$ and from 0.3 to $3.8 \mathrm{~g}$ of $\mathrm{EC} / \mathrm{kg}$ of flesh. The phenolic content observed in this study is comparable to that previously reported with the same methodology and standard for other plum varieties (3.0-5.6 g of 5CQA/kg) (30). Considering the results obtained on a 5CQA fresh weight basis: (i) in the flesh, GG-V shows the highest amount of phenolic compounds, with about $25 \%$ more than in GG-C and about 2-3fold more than in GJ, GG-F, and M; (ii) in the skin, GG-V and GG-C show the highest and a similar amount of phenolic compounds, with about 1.5-fold more than in GJ and 2-3-fold more than in GG-F and M. These results indicate the much higher amount of phenolic compounds present on the GG variety from Portugal from both orchards.

Quantification and Characterization of Phenolic Compounds by RP-HPLC. RP-HPLC with photodiode array detection was used to separate, characterize, and quantify the different classes of phenolic compounds present in plums. An example of the RP-HPLC chromatograms obtained for skin before and after thiolysis, with the attribution of the main components to the peaks, is presented in Figure 2. For flesh 
Table 3. Quantification of Phenolic Compounds in Plum Skin (mg/kg of Fresh Weight and mg/Fruit) by RP-HPLC, for Crude Extract and after Thiolysis ${ }^{a}$

\begin{tabular}{|c|c|c|c|c|c|c|c|c|c|c|}
\hline & \multicolumn{2}{|c|}{ GG-V } & \multicolumn{2}{|c|}{ GG-C } & \multicolumn{2}{|c|}{ GG-F } & \multicolumn{2}{|c|}{ M } & \multicolumn{2}{|c|}{ GJ } \\
\hline \multicolumn{11}{|c|}{ Hydroxycinnamic Acids } \\
\hline 5CQA (Rt $15.5 \mathrm{~min})$ & $722 \pm 19$ & 4 & $603 \pm 9$ & 4 & $544 \pm 5$ & 2 & $869 \pm 18$ & 1 & $77 \pm 5$ & 1 \\
\hline $\mathrm{FQA}^{b}$ (Rt $\left.15.0 \mathrm{~min}\right)$ & & & & & & & $40 \pm 2$ & & & \\
\hline $\mathrm{CSA}^{b}$ (Rt $\left.13.9 \mathrm{~min}\right)$ & $137 \pm 6$ & 1 & $130 \pm 5$ & 1 & $218 \pm 2$ & 1 & $74 \pm 2$ & $\operatorname{tr}$ & $69 \pm 2$ & 1 \\
\hline unknown (Rt $9.3 \mathrm{~min}$ ) & $87 \pm 3$ & 1 & $81 \pm 5$ & 1 & $133 \pm 1$ & 1 & $66 \pm 3$ & $\operatorname{tr}$ & $31 \pm 1$ & 1 \\
\hline unknown (Rt $20.5 \mathrm{~min}$ ) & $44 \pm 0$ & $\operatorname{tr}$ & $47 \pm 4$ & $\operatorname{tr}$ & & & & & & \\
\hline unknown (Rt $22.3 \mathrm{~min}$ ) & $42 \pm 10$ & tr & $44 \pm 4$ & $\operatorname{tr}$ & $63 \pm 0$ & tr & $42 \pm 1$ & $\operatorname{tr}$ & & \\
\hline subtotal & 4352 & 27 & 4196 & 25 & 4719 & 20 & 2601 & 3 & 612 & 9 \\
\hline \multicolumn{11}{|c|}{ Flavan-3-ols } \\
\hline B1 (Rt 13.4 min) & $247 \pm 6$ & 1 & $232 \pm 5$ & 1 & $117 \pm 4$ & 1 & $164 \pm 15$ & $\operatorname{tr}$ & $879 \pm 10$ & 12 \\
\hline CAT (Rt $14.8 \mathrm{~min}$ ) & $178 \pm 5$ & 1 & $184 \pm 2$ & 1 & $114 \pm 1$ & 1 & $117 \pm 5$ & $\operatorname{tr}$ & $1044 \pm 26$ & 15 \\
\hline unknown (Rt $12.7 \mathrm{~min}$ ) & $28 \pm 1$ & $\operatorname{tr}$ & $29 \pm 1$ & $\operatorname{tr}$ & & & $107 \pm 8$ & $\operatorname{tr}$ & $110 \pm 7$ & 2 \\
\hline unknown (Rt $15.8 \mathrm{~min}$ ) & $50 \pm 6$ & tr & $46 \pm 1$ & tr & & & & & $326 \pm 8$ & 5 \\
\hline unknown (Rt $16.8 \mathrm{~min}$ ) & $43 \pm 3$ & tr & $43 \pm 1$ & tr & & & & & $56 \pm 8$ & 1 \\
\hline unknown (Rt $18.9 \mathrm{~min}$ ) & $45 \pm 4$ & tr & $44 \pm 2$ & $\operatorname{tr}$ & & & & & $19 \pm 1$ & $\operatorname{tr}$ \\
\hline unknown (Rt $20.2 \mathrm{~min}$ ) & $52 \pm 6$ & $\operatorname{tr}$ & $51 \pm 2$ & $\operatorname{tr}$ & & & & & $259 \pm 15$ & 4 \\
\hline unknown (Rt $21.1 \mathrm{~min}$ ) & $53 \pm 2$ & tr & $55 \pm 2$ & $\operatorname{tr}$ & $63 \pm 1$ & $\operatorname{tr}$ & $54 \pm 1$ & $\operatorname{tr}$ & $22 \pm 1$ & tr \\
\hline unknown (Rt $23.3 \mathrm{~min}$ ) & $96 \pm 1$ & 1 & $83 \pm 9$ & 1 & & & & & $140 \pm 12$ & 2 \\
\hline subtotal & 1034 & 6 & 993 & 6 & 458 & 2 & 442 & 1 & 3595 & 52 \\
\hline \multicolumn{11}{|c|}{ Flavonols } \\
\hline Myr (Rt $22.7 \mathrm{~min})$ & $154 \pm 5$ & 1 & $110 \pm 2$ & 1 & $146 \pm 41$ & 1 & $268 \pm 30$ & $\operatorname{tr}$ & & \\
\hline Rut (Rt $22.9 \mathrm{~min}$ ) & $188 \pm 2$ & 1 & $110 \pm 3$ & 1 & $160 \pm 1$ & 1 & $436 \pm 12$ & 1 & & \\
\hline Hyper (Rt $23.2 \mathrm{~min}$ ) & & & & & & & & & $29 \pm 4$ & $\operatorname{tr}$ \\
\hline Isoquer (Rt $23.5 \mathrm{~min}$ ) & $49 \pm 1$ & $\operatorname{tr}$ & $43 \pm 3$ & $\operatorname{tr}$ & $40 \pm 5$ & $\operatorname{tr}$ & $133 \pm 3$ & $\operatorname{tr}$ & $27 \pm 2$ & tr \\
\hline \multicolumn{11}{|c|}{ Procyanidins } \\
\hline EC & $231 \pm 12$ & 1 & $234 \pm 7$ & 1 & $198 \pm 15$ & 1 & $127 \pm 1$ & tr & $103 \pm 4$ & 1 \\
\hline A & & & & & & & & & $605 \pm 20$ & 8 \\
\hline EC-Ext (Rt 36.0 min) & $2904 \pm 128$ & 17 & $3204 \pm 66$ & 19 & $2155 \pm 0$ & 9 & $878 \pm 76$ & 1 & $2316 \pm 21$ & 32 \\
\hline unknown (Rt $23.9 \mathrm{~min}$ ) & $69 \pm 2$ & 23 & & & $101 \pm 5$ & 1 & & & $98 \pm 5$ & 1 \\
\hline subtotal & 3840 & 45 & 4203 & 25 & 2994 & 13 & 1289 & 1 & 4566 & 62 \\
\hline total & 8685 & 75 & 8735 & 52 & 8188 & 35 & 4828 & 5 & 5400 & 74 \\
\hline DPn & \multicolumn{2}{|c|}{$5.8 \pm 0.1$} & \multicolumn{2}{|c|}{$5.6 \pm 0.1$} & \multicolumn{2}{|c|}{$5.1 \pm 0.4$} & \multicolumn{2}{|c|}{$4.0 \pm 0.1$} & \multicolumn{2}{|c|}{$5.5 \pm 0.2$} \\
\hline
\end{tabular}

${ }^{a}$ Flavan-3-ols are expressed as catechin equivalents; hydroxycinnamic acids are expressed as 5-caffeoylquinic acid equivalents; and flavonols are expressed as rutin equivalents. A, A-type procyanidin; B1, procyanidin B1; B2, procyanidin B2; B7, procyanidin B7; CAT, (+)-catechin; 3CQA, 3-caffeoylquinic acid; 5CQA, 5-caffeoylquinic acid; CSA, caffeoylshikimic acid; DPn, average degree of polymerization; EC, (-)-epicatechin; EC-Ext, (-)-epicatechin benzylthioether; FQA, feruloylquinic acid; GG-C, 'Green Gage'-Portugal "Cano”; GG-F, 'Green Gage'-France; GG-V, 'Green Gage'-Portugal "Vila Viçosa”; GJ, 'Golden Japan'; Hyper, hyperoside; Isoquer, isoquercitrin; Isorham-rut, isorhamnetin rutinoside; M, 'Mirabelle'; Myr, myricetin; Quer, quercitrin; Rt, retention time; Rut, rutin; tr, trace amounts, $<0.5 \mathrm{mg} /$ fruit. Mean \pm standard deviation $(n=3) .{ }^{b}$ Identification only by ESI-MS, no standard available.

tissues, the chromatograms were similar, except that there are no peaks corresponding to flavonols.

In the crude extract without thiolysis, compounds of the procyanidin type appeared as more or less unresolved peaks. After thiolytic depolymerization, two kinds of compounds are released: catechin or epicatechin terminal units depending upon the nature of flavan-3-ol and flavanol units linked to benzyl thioether corresponding to extension units, giving well-resolved peaks that permitted accurate integration. As already mentioned for apple samples submitted to thiolysis (20), the thiolysis chromatograms showed additional peaks resulting from side reactions without significant consequences for quantification purposes: hydroxycinnamic acids are partly converted into their corresponding methylester, and flavonols are partly deglycosylated.

Phenolic Compounds in the Flesh. In the flesh of plum extracts, two classes of compounds, hydroxycinnamic acid derivatives and flavan-3-ols, were detected (Table 2). The main class of phenolic compounds quantified in the crude flesh extracts was the hydroxycinnamic acid derivatives. For the GG variety, the content of hydroxycinnamic acid derivatives ranged from 2.0 to $2.6 \mathrm{~g} / \mathrm{kg}$, which contrasts with the lower values for $\mathrm{M}(1.2 \mathrm{~g} / \mathrm{kg})$ and GJ $(0.2 \mathrm{~g} / \mathrm{kg})$. In all varieties, 3-caffeoylquinic acid was the main phenolic compound, followed by 5-caffeoylquinic acid (Table 2). In GG flesh samples, 3-caffeoylquinic acid accounted for $81-83 \%$ of total hydroxycinnamic acids, 

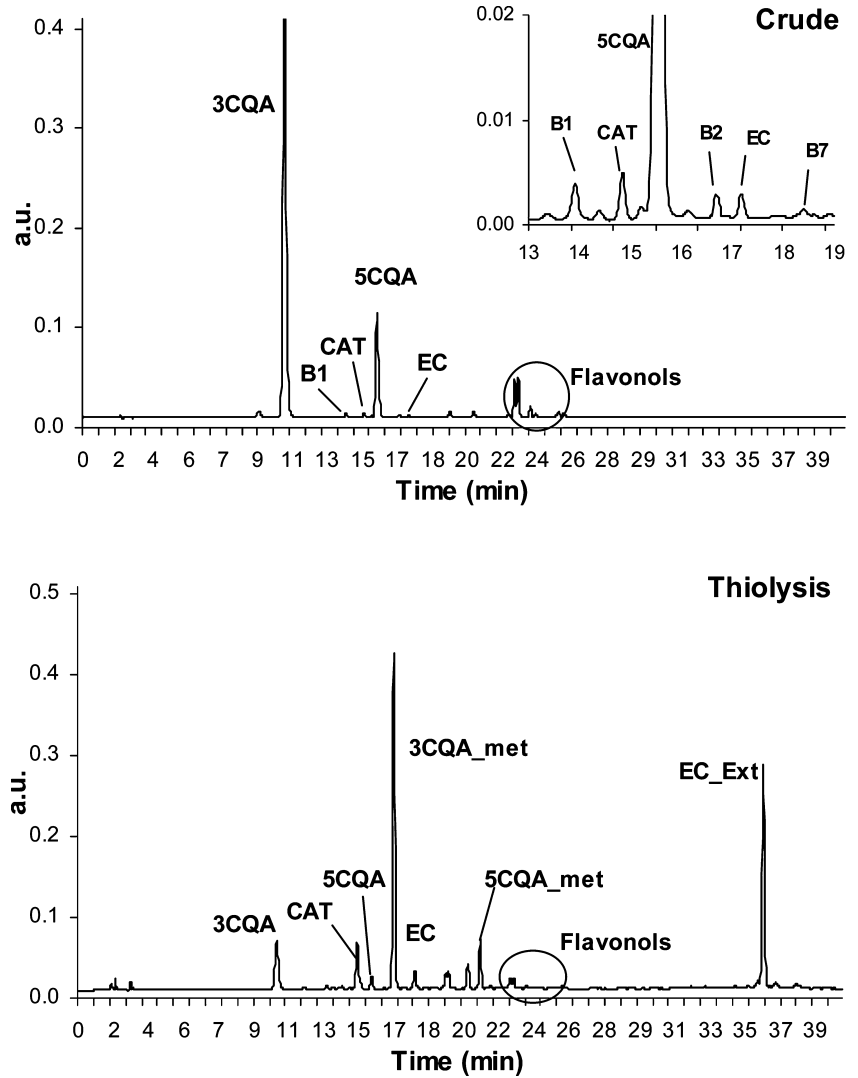

Figure 2. RP-HPLC chromatograms $(280 \mathrm{~nm})$ of the crude extract and after thiolysis of plums (GG-C) (B1, procyanidin B1; B2, procyanidin B2; $B 7$, procyanidin B7; CAT, (+)-catechin; 3CQA, 3-caffeoylquinic acid; 3CQA_met, 3-caffeoylquinic methyl ester; 5CQA, 5-caffeoylquinic acid; 5CQA_met, 5-caffeoylquinic methyl ester; EC, (-)-epicatechin; EC-Ext, (-)-epicatechin benzylthioether).

whereas in M and GJ, it represented slightly lower values (74 and $67 \%$ ). These hydroxycinnamic acid derivatives have been also observed in previous studies on plums and in similar quantities $(2,7,13-15,26,30)$. Although in small amounts, some other hydroxycinnamic acid derivatives were also detected by their characteristic UV spectra, which allowed their quantification by the 5 -caffeoylquinic acid standard. A caffeic acid derivative, with a $[\mathrm{M}-\mathrm{H}]^{-}$ion at $\mathrm{m} / \mathrm{z} 335$ and characteristic fragments at $\mathrm{m} / \mathrm{z} 179,161$, and 135, was also detected. It was identified as 3-O-caffeoylshikimic acid by a comparison to previously published MS data showing the same pseudomolecular ions and the same fragmentations for these compounds reported in dried plums (31). In addition, a 3-O-feruloylquinic acid was identified in M plums, with a MS spectrum that had a $[\mathrm{M}-\mathrm{H}]^{-}$ion at $\mathrm{m} / \mathrm{z}, 367$, in accordance with its molecular weight, and a fragment at $\mathrm{m} / \mathrm{z} 193$ that corresponded to the loss of the quinic acid moiety by MS fragmentation of the ester linkage (31). No evidence were obtained in the MS spectra for the presence of diCQA, neither for the corresponding $p$-coumaric and ferulic derivatives of shikimic acid.

Procyanidin B1 and (+)-catechin were the flavan-3-ols present in higher amount in all samples (Table 2). The flavan-3-ol monomers (-)-epicatechin and ( + -)-catechin were detected in the crude extracts for GG-C, GG-V, and GJ samples, with (+)catechin being highly preponderant, while in $\mathrm{M}$ and GG-F samples, only $(+)$-catechin was identified. Other studies of plums also describe $(+)$-catechin as the main monomeric flavan3-ol detected, with (-)-epicatechin being present in smaller amounts (13-15). Procyanidin B7 [dimer of (-)-epicatechin and $(+)$-catechin with a C4-C6 linkage] was identified only in the three samples of the GG variety. The procyanidin dimers of B-type were identified by a retention time comparison in a HPLC - photodiode array with the authentic markers available and by their characteristic ESI-MS spectra $(\mathrm{m} / \mathrm{z}, 577$, [M $\mathrm{H}]^{-} ; \mathrm{m} / z$ 425, retro-Diels-Alder fragment; and $\mathrm{m} / z, 289$ and 287, fragments of terminal and extension (+)-catechin residues in the dimer) $(14,32,33)$. Other procyanidin dimers and trimers (characteristic fragments at $\mathrm{m} / \mathrm{z} 865,577$, and 289) were also detected. Although they could not be fully identified, they were quantified as (+)-catechin equivalents using the corresponding calibration curve.

While the main class of phenolic compounds quantified in the crude extract was the hydroxycinnamic acid derivatives for GG $(72-91 \%$ of total phenolics quantified in crude extract) and M (88\%) varieties, GJ showed a very different phenolic composition. For this plum, the class of flavan-3-ols was the main class of phenolic compounds (85\% of total), B1 was the major procyanidin identified, and an A-type procyanidin dimer was only identified in this plum. The A-type procyanidin dimer detected (characteristic MS fragments at $\mathrm{m} / \mathrm{z} 575,[\mathrm{M}-\mathrm{H}]^{-}$; $\mathrm{m} / \mathrm{z}, 423$, retro-Diels-Alder fragment; and $\mathrm{m} / \mathrm{z}, 287$ and 285) showed an ESI-MS spectra similar to those of the B-type dimers but with two mass units less, as expected for this type of molecule with two intercatechin bonds. Procyanidin dimers (B1, B2, and A-type dimers) have already been described as the main phenolic compounds in the flesh of other plums, although in smaller amounts than observed for GJ plums (13). This work also confirms the occurrence of B7 dimers previously reported to be present in plums (34).

Because the thiolysis reaction causes depolymerization, it gives access to the RP-HPLC quantification of the part of procyanidins that are not accessible to quantification when the crude extract was analyzed. As a consequence, on the basis of RP-HPLC analysis of the thiolysed samples (Table 2), the amount of phenolic compounds quantified was higher than in crude extracts for all varieties (about 2-fold), indicating the presence of high concentrations of procyanidins. As observed for the crude extract, plums of the 'Green Gage' variety (GG$\mathrm{V}$, GG-C, and GG-F) presented the highest phenolic content, when compared to $\mathrm{M}$ and GJ. The amount of phenolics of these plums after thiolysis ranged from $5.6 \mathrm{~g} / \mathrm{kg}$ of fresh weight for GG-C to $2.7 \mathrm{~g} / \mathrm{kg}$ for GJ, which shows that some of these varieties, namely, GG, are very rich in phenolic compounds. On a fruit basis, the amount of phenolic compounds ranged from 146 and $124 \mathrm{mg} /$ fruit for GG-V and GG-C, respectively, to 26 $\mathrm{mg} /$ fruit for M. On a fruit basis, the Portuguese 'Green Gage' plums and GJ plums, the biggest one, were the richest when compared to $\mathrm{M}$ variety and GG-F (Table 2). The average quantity of phenolic compounds calculated after RP-HPLC analysis in other studies for the plum flesh was usually lower, $1.1(1,12,14), 2.8(13), 2.7(26)$, and $3.7 \mathrm{~g} / \mathrm{kg}$ of fresh weight (15), probably because data presented here take into account the amount of polymerized procyanidins.

As expected, after thiolysis, an increase of total flavanols (i.e., the sum of $(+)$-catechin, $(-)$-epicatechin, and (-)-epicatechin benzylthioether) was observed. As a consequence, the class of flavan-3-ols became the main class of phenolic compounds identified for all samples analyzed, $92 \%$ in GJ, $62 \%$ in GG-C, $61 \%$ in GG-V, and $54 \%$ in M, except for GG-F (45\%). These results may explain the differences in the ratio between the values of total phenolics calculated using EC and 5CQA as standards observed by the Folin-Ciocalteu method, suggesting that the higher ratio EC/5CQA is related to a higher proportion of hydroxycinnamic acids in relation to flavan-3-ols in samples. 
Terminal units of procyanidins observed in plums are mainly $(+)$-catechin $(54-77 \%$ of all terminal units). In GJ, as previously observed in the pericarp of lichi fruits (28), A-type procyanidin was detected after thiolysis in a higher quantity than in crude extract, which suggests its presence as native dimers and also as terminal residues in the structure of procyanidin oligomers.

RP-HPLC analyses following thiolysis allows for the determination of the average DPn of procyanidins (bottom line in Table 2). The flesh of GG-V plums showed the highest polymerized procyanidins, with a DPn of approximately 8.5, followed by GG-C (DPn = 6.6). GG-F and M showed a DPn of 5.4 and 5.7, respectively, and the lowest DPn of flesh procyanidins was observed for GJ $(\mathrm{DPn}=4.5)$.

Phenolic Compounds in the Skin. The total content of phenolic compounds on a weight basis was much higher in the skin than in the flesh, in agreement with other studies in plums $(6,14,30)$. However, the contribution of the phenolic compounds of the skin to the overall content of phenolic compounds of the fruit is lower than that of the flesh (Tables 2 and 3). This is due to the lower proportion of skin weight when compared to the flesh of the fruit (Table 1), which ranged from $14,22-25$, and $38 \%$, respectively for $M$, the three $G G$, and GJ plums.

The main hydroxycinnamic acid derivatives and flavan-3ols detected and quantified in the flesh were also identified in the skin of the plums, as is shown in Table 3. As observed in the flesh, the main class of phenolic compounds quantified in the crude extract of the skin was the hydroxycinnamic acid derivatives ranging from 0.6 to $4.7 \mathrm{~g} / \mathrm{kg}$, except for GJ. For the latter variety, the main class of phenolic compounds was the flavan-3-ols ( $81 \%$ of total phenolic compounds), similarly to what was found for the flesh. Also, 3-caffeoylquinic acid was, by far, the most abundant hydroxycinnamic acid derivative, and the GG variety accounted for a higher percentage of this compound (75-77\% of total hydroxycinnamic acids). Procyanidin B1 and (+)-catechin were the flavan-3-ols present in higher amounts in all samples, in about similar proportions. As observed for the flesh, procyanidin B7 was found in GG-V and GG-C samples, although not in GG-F, and A-type procyanidin was only found in GJ.

In the skin, another class of phenolic compounds, the flavonols, was detected. For crude methanol extract, they accounted for $8 \%$ or less of the total phenolics, except for the $\mathrm{M}$ variety, for which they represented $24 \%$ (Table 3). Similar relative amounts of flavonols, as those occurring in the $\mathrm{M}$ variety, have been also reported for other plums varieties (e.g., 'Autumn Sweet', 'Beltsville Elite', 'B70197', 'Castleton', 'Empress', 'Longjohn', and 'Stanley') (26). The main flavonol compound was rutin (rhamnoglucoside of quercetin), which accounted for $0.4 \mathrm{~g} / \mathrm{kg}$ of skin in $\mathrm{M}$ and $0.1-0.2 \mathrm{~g} / \mathrm{kg}$ of skin in GG varieties, followed by myricetin, although these two flavonols have not been identified in GJ. In addition, quercitrin and hyperoside were only identified in GJ plums. Previous studies on plums also showed rutin as the principal flavonol, ranging from 10 to $100 \mathrm{mg} / \mathrm{kg}(7,13,14,26,30)$. Various quercetin glycosides, as mono- or disaccharides, were previously found in plums, such as quercetin glucoside (isoquercitrin), quercetin rhamnoside (quercitrin), and quercetin xyloside and, in smaller amounts, kaempferol 3-rutinoside, 3-glucoside, 3-galactoside, and 3-arabinoside-7-rhamnoside $(1,12,13)$. The total amount of flavonols that have been reported to be present in plum skin ranged from 30 to $350 \mathrm{mg} / \mathrm{kg}$ of fresh weight, depending upon the variety $(12,13,26)$. The occurrence of these flavonols in higher quantities in the varieties under study should be exploited, because some of these compounds, such as quercetin glycosides, show relevant biological activities (19).

After thiolysis, the total amount of phenolic compounds increased but to a lesser extent compared to the flesh, being noticeable only for the three GG samples and, among these, more substantially for GG-V and GG-C. This indicates a possible presence of lower proportions of procyanidins in the skin, when compared to the flesh. Flavan-3-ols accounted for $85 \%$ of total phenolic compounds in the skin of GJ, $48 \%$ in GG-C, $44 \%$ in GG-V, $37 \%$ in GG-F, and $27 \%$ in M. The total amount of phenolic compounds quantified by RP-HPLC in skin ranged from $8.7 \mathrm{~g} / \mathrm{kg}$ of fresh weight for GG-C and GG-V to $4.8 \mathrm{~g} / \mathrm{kg}$ for M. As it was observed for the flesh, the Portuguese 'Green Gage' plums were richer $(p<0.05)$ in phenolic compounds when compared to GG-F $(8.2 \mathrm{~g} / \mathrm{kg})$ and the other plums analyzed. The A-type procyanidin of GJ increased about 2-fold with thiolysis, which means that this dimer is present in GJ skin and flesh as a native and terminal unit of the procyanidin oligomers.

As observed in the flesh, the GG-V and GG-C plums presented the highest polymerized procyanidins in the skin, with a DPn of 5.8 and 5.6, respectively (bottom line in Table 3), which however were lower than that of the flesh. The procyanidins of GG-F skin had a similar DPn (5.1) to those present in the flesh, and the procyanidins of GJ showed a slightly higher DPn than those of the flesh $(\mathrm{DPn}=5.5)$.

PCA. A comparative analysis of the phenolic compounds present in the five samples studied was performed by means of PCA both in the flesh (Figure 3) and in the skin (Figure 4) as an attempt to identify the different plums based on their phenolic compound contents.

Figure 3a shows the scores scatter plot $(\mathrm{PC} 1 \times \mathrm{PC} 2)$ of the data provided by the analysis of phenolic compounds of the flesh, where four groups were obtained. This analysis allows for the differentiation of the Portuguese GG samples (PC1 positive and PC2 negative), GG-F (PC2 positive), M (PC1 negative and PC2 positive), and GJ (PC1 and PC2 negative). These two axes contain $99 \%$ of the total variability. According to the loadings plot (parts $\mathbf{b}$ and $\mathbf{c}$ of Figure 3), the distinction of the GG samples is due to the higher content of hydroxycinnamic acids, mainly 3-caffeoylquinic acid, and the flavan-3ols, mostly the procyanidins. GG-C and GG-V are distinguished from GG-F because of the higher content of procyanidins in Portuguese GG samples. GJ plum is distinguished from the other varieties as a result of the presence of A-type procyanidin, procyanidin $\mathrm{B} 1$, and $(+)$-catechin.

The PC1 $\times$ PC2 scores scatter plot of phenolic compounds present in skin distinguished the GG varieties (PC1 and PC2 positive) from $\mathrm{M}$ (PC1 positive and PC2 negative) and GJ (PC1 and PC2 negative) (results not shown). The loadings plots (PC1 results not shown) demonstrated that GG plums were separated according to their content in 3-caffeoylquinic acid, procyanidin B7, (-)-epicatechin terminally linked to procyanidins, and the flavonol isorhamnetin rutinoside. The PC2 $\times$ PC3 scores scatter plot (Figure 4a) distinguished the Portuguese GG variety (PC2 positive and PC3 negative) from GG-F (PC2 and PC3 positive) and $\mathrm{M}$ and GJ (PC2 and PC3 negative). According to the loadings plot (parts $\mathbf{b}$ and $\mathbf{c}$ of Figure 4), the distinction of the GG samples from the others is due to the higher content of procyanidins, namely, the oligomeric (-)-epicatechin and (+)catechin, and the dimers B2 and B7. GG-C and GG-V are distinguished from GG-F as a result of the presence of 


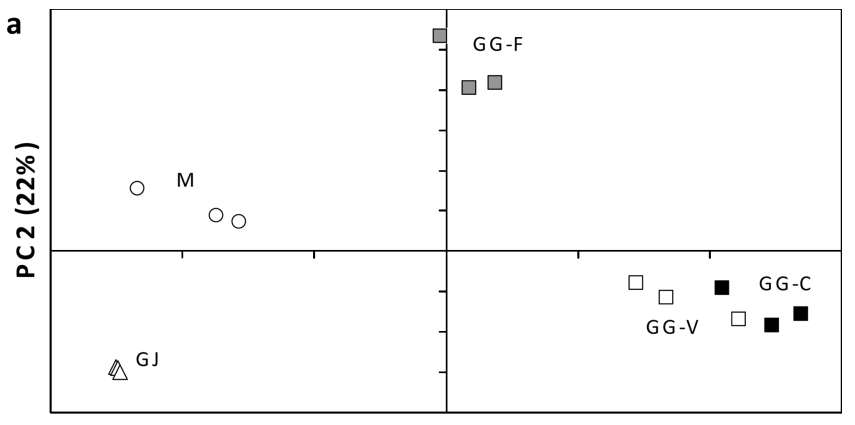

PC 1 (77\%)

$\square G G-V \quad \square G G-C \quad \square G G-F \quad O M \quad \triangle G J$
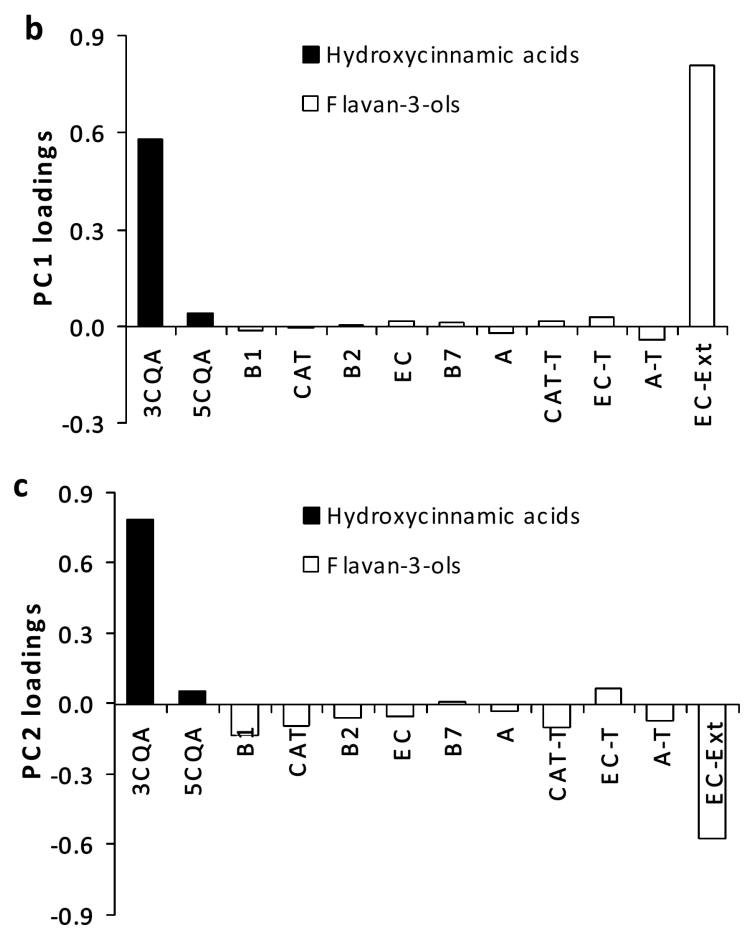

Figure 3. PCA of the phenolic compounds of plum flesh: (a) scores scatter plot (PC1 versus PC2) and (b) PC1 and (c) PC2 loadings. A, A-type dimeric procyanidin; A-T, A-type terminal dimeric procyanidin; B1, procyanidin $\mathrm{B} 1$; $\mathrm{B} 2$, procyanidin $\mathrm{B} 2$; $\mathrm{B} 7$, procyanidin $\mathrm{B} 7$; $\mathrm{CAT}$, monomeric (+)-catechin; CAT-T, terminal (+)-catechin; 3CQA, 3-caffeoylquinic acid; 5CQA, 5-caffeoylquinic acid; EC, monomeric (-)-epicatechin; EC-Ext, extension (-)-epicatechin; EC-T, terminal (-)-epicatechin.

procyanidin B7 in GG-V and GG-C and not in GG-F skin and the higher content of oligomeric procyanidins.

The data obtained by thiolysis followed by RP-HPLC and chemometrics of the plum phenolic compounds allowed us to observe that the plums from different variety and species and even plums of the same variety but from different origins could be distinguished according to their characteristic phenolic composition of the flesh and/or skin. These diagnostic compounds identified here should however be confirmed using for a comparison other varieties, harvests, and stages of ripening. Anyway, they emphasize the importance of the geographical local of production (resulting in possible different production procedures and climate influences) for the total phenolic content of plums.

\section{ABBREVIATIONS USED}

A, procyanidin A; A-T, procyanidin A terminal dimer; B1, procyanidin $\mathrm{B} 1$; B2, procyanidin $\mathrm{B} 2$; B7, procyanidin $\mathrm{B} 7$; CAT,
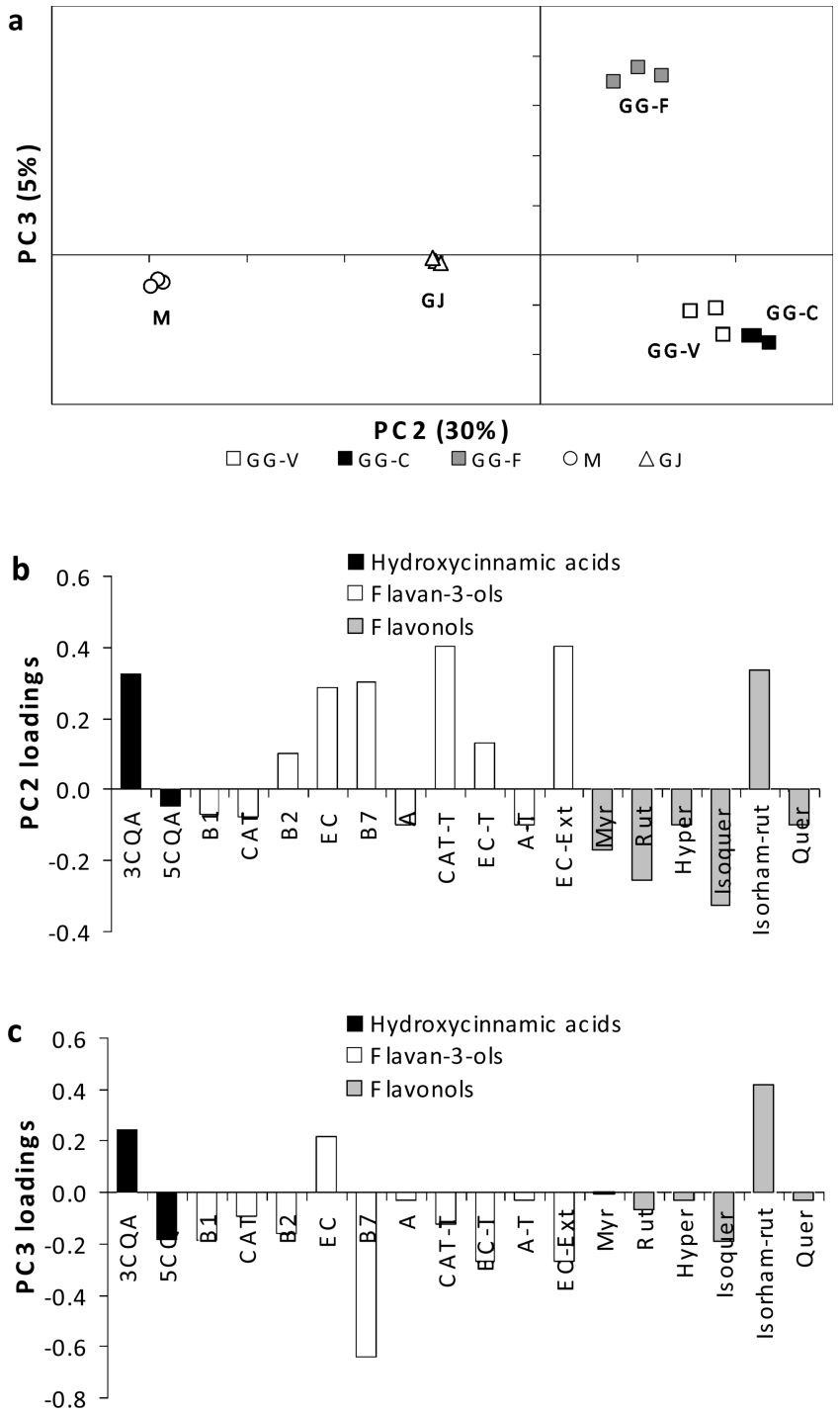

Figure 4. PCA of the phenolic compounds of plum skin: (a) scores scatter plot (PC2 versus PC3) and (b) PC2 and (c) PC3 loadings. A, A-type dimeric procyanidin; A-T, A-type terminal dimeric procyanidin; B1, procyanidin $\mathrm{B} 1$; $\mathrm{B} 2$, procyanidin $\mathrm{B} 2$; $\mathrm{B}$ 7, procyanidin $\mathrm{B} 7$; $\mathrm{CAT}$, monomeric $(+)$-catechin; CAT-T, terminal (+)-catechin; 3CQA, 3-caffeoylquinic acid; 5CQA, 5-caffeoylquinic acid; EC, monomeric (-)-epicatechin; EC-Ext, extension (-)-epicatechin; EC-T, terminal (-)-epicatechin; Hyper, hyperoside; Isoquer, isoquercitrin; Isorham-rut, isorhamnetin rutinoside; Myr, myricetin; Quer, quercitrin; Rut, rutin.

(+)-catechin; CAT-T, (+)-catechin terminal units; 3CQA, 3-caffeoylquinic acid; 3CQA_met, 3-caffeoylquinic methyl ester; 5CQA, 5-caffeoylquinic acid; 5CQA_met, 5-caffeoylquinic methyl ester; CSA, caffeoylshikimic acid; DPn, average degree of polymerization; EC, (-)-epicatechin; EC-Ext, (-)-epicatechin benzylthioether; EC-T, (-)-epicatechin terminal units; ESI-MS, electrospray ionization-mass spectrometry; FQA, feruloylquinic acid; GG-C, 'Green Gage'-Portugal 'Cano'; GG-F, 'Green Gage'-France; GG-V, 'Green Gage'-Portugal 'Vila Viçosa'; GJ, 'Golden Japan'; Hyper, Hyperoside; Isoquer, isoquercitrin; Isorham-rut, isorhamnetin rutinoside; M, 'Mirabelle'; Myr, myricetin; Quer, Quercitrin; RP-HPLC, reversed-phase highperformance liquid chromatography; Rut, rutin.

\section{ACKNOWLEDGMENT}

We thank Confibor Lda, Estremoz, Portugal, for providing the samples and Prof. João Mota Barroso and Mrs. Ana Elisa 
Rato, from Universidade de Évora, Portugal, for helpful discussions about plums. Cláudia Nunes thanks FCT (Portugal) for the Ph.D. Grant (SFRH/BD/12928/2003) and Programa PESSOA ( Proc $^{\circ} 4.1 .1 /$ França). Thanks are also due to Project AGRO 220 and FCT, Portugal, for funding the Research Unit 62/94 'Química Orgânica, Produtos Naturais e Agro-Alimentares'.

\section{LITERATURE CITED}

(1) Cheynier, V. Polyphenols in foods are more complex than often thought. Am. J. Clin. Nutr. 2005, 81 (1), 223S-229S.

(2) Kim, D.-O.; Jeong, S. W.; Lee, C. Y. Antioxidant capacity of phenolic phytochemicals from various cultivars of plums. Food Chem. 2003, 81 (3), 321-326.

(3) Gil, M. I.; Tomas-Barberan, F. A.; Hess-Pierce, B.; Kader, A. A. Antioxidant capacities, phenolic compounds, carotenoids, and vitamin $\mathrm{C}$ contents of nectarine, peach, and plum cultivars from California. J. Agric. Food Chem. 2002, 50 (17), 4976-4982.

(4) Kim, D. O.; Lee, K. W.; Chun, O. K.; Leer, H. J.; Lee, C. Y. Antiproliferative activity of polyphenolics in plums. Food Sci. Biotechnol. 2003, 12 (4), 399-402.

(5) Bengoechea, M. L.; Sancho, A. I.; Bartolome, B.; Estrella, I.; Gomez Cordoves, C.; Hernandez, M. T. Phenolic composition of industrially manufactured purees and concentrates from peach and apple fruits. J. Agric. Food Chem. 1997, 45 (10), 4071-4075.

(6) Imeh, U.; Khokhar, S. Distribution of conjugated and free phenols in fruits: Antioxidant activity and cultivar variations. J. Agric. Food Chem. 2002, 50 (22), 6301-6306.

(7) Chun, O. K.; Kim, D. O.; Moon, H. Y.; Kang, H. G.; Lee, C. Y. Contribution of individual polyphenolics to total antioxidant capacity of plums. J. Agric. Food Chem. 2003, 51 (25), 72407245 .

(8) Wang, H.; Cao, G. H.; Prior, R. L. Total antioxidant capacity of fruits. J. Agric. Food Chem. 1996, 44 (3), 701-705.

(9) Murcia, M. A.; Jimenez, A. M.; Martinez-Tome, M. Evaluation of the antioxidant properties of Mediterranean and tropical fruits compared with common food additives. J. Food Prot. 2001, 64 (12), 2037-2046.

(10) Nakatani, N.; Kayano, S.; Kikuzaki, H.; Sumino, K.; Katagiri, K.; Mitani, T. Identification, quantitative determination, and antioxidative activities of chlorogenic acid isomers in prune (Prunus domestica L.). J. Agric. Food Chem. 2000, 48 (11), 55125516.

(11) Rupasinghe, H. P. V.; Jayasankar, S.; Lay, W. Variation in total phenolics and antioxidant capacity among European plum genotypes. Sci. Hortic. 2006, 108 (3), 243-246.

(12) Lombardi-Boccia, G.; Lucarini, M.; Lanzi, S.; Aguzzi, A.; Cappelloni, M. Nutrients and antioxidant molecules in yellow plums (Prunus domestica L.) from conventional and organic productions: A comparative study. J. Agric. Food Chem. 2004, 52 (1), 90-94.

(13) Tomás-Barberán, F. A.; Gil, M. I.; Cremin, P.; Waterhouse, A. L.; Hess-Pierce, B.; Kader, A. A. HPLC-DAD-ESIMS analysis of phenolic compounds in nectarines, peaches, and plums. J. Agric. Food Chem. 2001, 49 (10), 4748-4760.

(14) Donovan, J. L.; Meyer, A. S.; Waterhouse, A. L. Phenolic composition and antioxidant activity of prunes and prune juice (Prunus domestica). J. Agric. Food Chem. 1998, 46 (4), 12471252.

(15) Piga, A.; Del Caro, A.; Corda, G. From plums to prunes: Influence of drying parameters on polyphenols and antioxidant activity. $J$. Agric. Food Chem. 2003, 51 (12), 3675-3681.

(16) Guyot, S.; Marnet, N.; Drilleau, J. F. Thiolysis-HPLC characterization of apple procyanidins covering a large range of polymerization states. J. Agric. Food Chem. 2001, 49 (1), 14-20.

(17) Scalbert, A. Antimicrobial properties of tannins. Phytochemistry 1991, 30, 3875-3883.
(18) Rios, L. Y.; Bennett, R. N.; Lazarus, S. A.; Rémésy, C.; Scalbert, A.; Williamson, G. Cocoa procyanidins are stable during gastric transit in humans. Am. J. Clin. Nutr. 2002, 76, 1106-1110.

(19) Kahle, K.; Huemmer, W.; Kempf, M.; Scheppach, W.; Erk, T.; Richling, E. Polyphenols are intensively metabolized in the human gastrointestinal tract after apple juice consumption. J. Agric. Food Chem. 2007, 55 (26), 10605-10614.

(20) Guyot, S.; Marnet, N.; Laraba, D.; Sanoner, P.; Drilleau, J. F. Reversed-phase HPLC following thiolysis for quantitative estimation and characterization of the four main classes of phenolic compounds in different tissue zones of a French cider apple variety (Malus domestica var. Kermerrien). J. Agric. Food Chem. 1998, 46 (5), 1698-1705.

(21) Hemingway, R. W.; McGraw, G. W. Kinetics of acid-catalyzed cleavage of procyanidins. J. Wood Chem. Technol. 1983, 3 (4), 421-435.

(22) Kim, D.-O.; Lee, C. Y. Extraction and isolation of polyphenolics. In Current Protocols in Food Analytical Chemistry; Wrolstad, R. E., Ed.; Wiley: New York, 2002; pp I1.2.1-I1.2.12.

(23) Singleton, V. L.; Orthofer, R.; Lamuela-Raventós, R. M. Analysis of total phenols and other oxidation substrates and antioxidants by means of Folin-Ciocalteu reagent. In Methods in Enzymology, 1st ed.; Sies, H., Abelson, J., Simon, M., Eds.; Academic Press (Elsevier, Inc.): Burlington, MA, 1999; Vol. 299, pp 152-178.

(24) Chun, O. K.; Kim, D. O. Consideration on equivalent chemicals in total phenolic assay of chlorogenic acid-rich plums. Food Res. Int. 2004, 37 (4), 337-342.

(25) Ferreira, D.; Guyot, S.; Marnet, N.; Delgadillo, I.; Renard, C.; Coimbra, M. A. Composition of phenolic compounds in a Portuguese pear (Pyrus communis L. var. S. Bartolomeu) and changes after sun-drying. J. Agric. Food Chem. 2002, 50 (16), 4537-4544.

(26) Kim, D. O.; Chun, O. K.; Kim, Y. J.; Moon, H. Y.; Lee, C. Y. Quantification of polyphenolics and their antioxidant capacity in fresh plums. J. Agric. Food Chem. 2003, 51 (22), 6509-6515.

(27) Delage, E.; Bohuon, G.; Baron, A.; Drilleau, J. F. Highperformance liquid chromatography of the phenolic compounds in the juice of some french cider apple varieties. J. Chromatogr. 1991, 555 (1-2), 125-136.

(28) Le Roux, E.; Doco, T.; Sarni-Manchado, P.; Lozano, Y.; Cheynier, V. A-type proanthocyanidins from pericarp of Litchi chinensis. Phytochemistry 1998, 48 (7), 1251-1258.

(29) Jolliffe, I. T. Principal Component Analysis, 2nd ed.; Springer: New York, 2004.

(30) Cevallos-Casals, B. A.; Byrne, D.; Okie, W. R.; Cisneros-Zevallos, L. Selecting new peach and plum genotypes rich in phenolic compounds and enhanced functional properties. Food Chem. 2006, 96 (2), 273-280.

(31) Fang, N. B.; Yu, S. G.; Prior, R. L. LC/MS/MS characterization of phenolic constituents in dried plums. J. Agric. Food Chem. 2002, 50 (12), 3579-3585.

(32) Friedrich, W.; Eberhardt, A.; Galensa, R. Investigation of proanthocyanidins by HPLC with electrospray ionization mass spectrometry. Eur. Food Res. Technol. 2000, 211, 56-64.

(33) Hammerstone, J. F.; Lazarus, S. A.; Mitchell, A. E.; Rucker, R.; Schmitz, H. H. Identification of procyanidins in cocoa (Theobroma cacao) and chocolate using high-performance liquid chromatography mass spectrometry. J. Agric. Food Chem. 1999, 47 (2), 490496.

(34) Pascual-Teresa, S.; Santos-Buelga, C.; Rivas-Gonzalo, J. C. Quantitative analysis of flavan-3-ols in Spanish foodstuffs and beverages. J. Agric. Food Chem. 2000, 48 (11), 5331-5337.

Received for review December 28, 2007. Revised manuscript received April 3, 2008. Accepted April 10, 2008.

JF8006135 